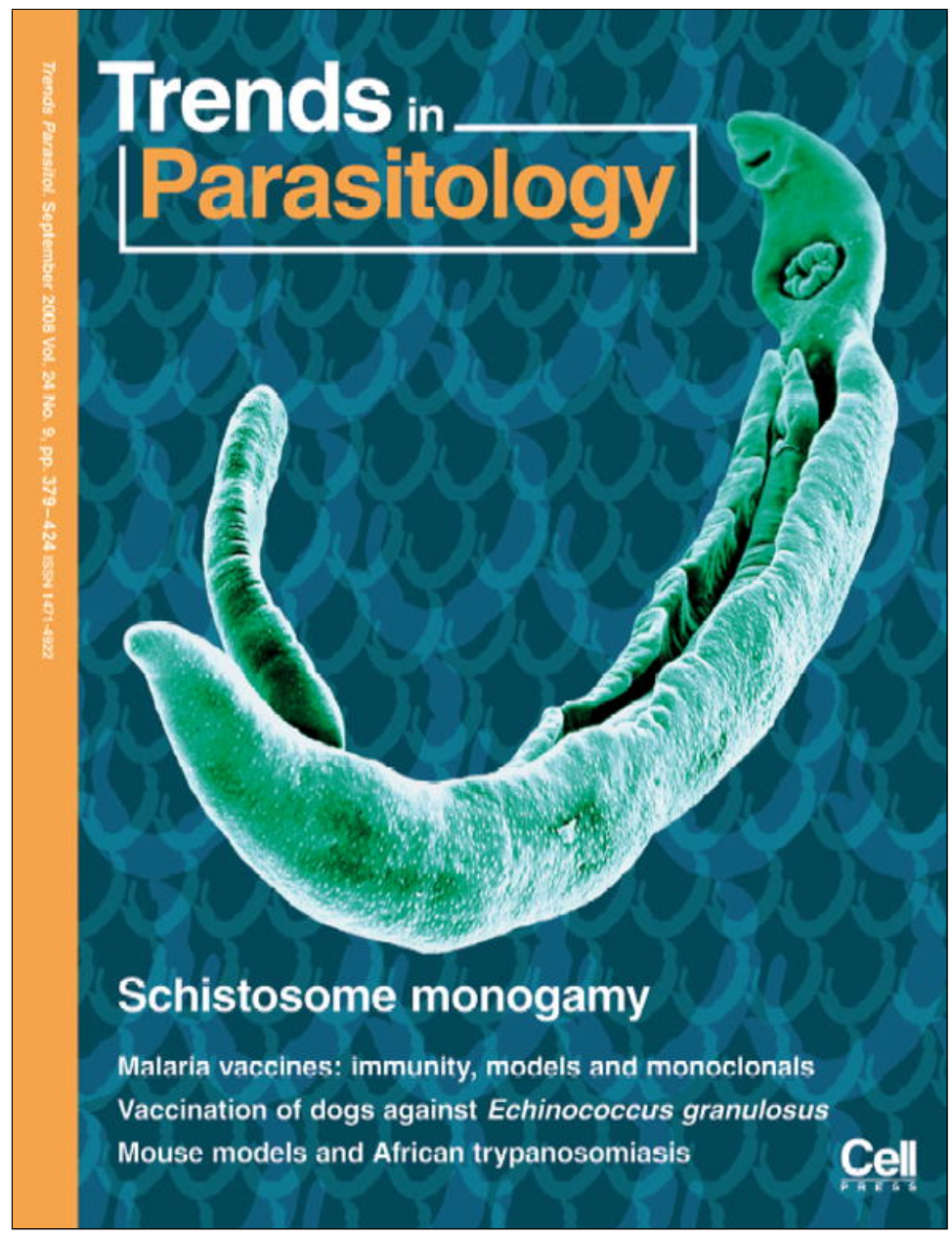

This article appeared in a journal published by Elsevier. The attached copy is furnished to the author for internal non-commercial research and education use, including for instruction at the authors institution and sharing with colleagues.

Other uses, including reproduction and distribution, or selling or licensing copies, or posting to personal, institutional or third party websites are prohibited.

In most cases authors are permitted to post their version of the article (e.g. in Word or Tex form) to their personal website or institutional repository. Authors requiring further information regarding Elsevier's archiving and manuscript policies are encouraged to visit:

http://www.elsevier.com/copyright 


\title{
Contributions of experimental mouse models to the understanding of African trypanosomiasis
}

\author{
Nicolas Antoine-Moussiaux ${ }^{1,2}$, Stefan Magez ${ }^{3,4}$ and Daniel Desmecht ${ }^{1}$ \\ ${ }_{1}^{1}$ Department of Animal Pathology, Veterinary College, University of Liege, 20 Boulevard de Colonster, B43, 4000 Liege, Belgium \\ ${ }^{2}$ Tropical Veterinary Institute, Veterinary College, University of Liege, 20 Boulevard de Colonster, B43, 4000 Liege, Belgium \\ ${ }^{3}$ Department of Molecular and Cellular Interactions, Flanders Institute for Biotechnology (VIB), Pleinlaan 2, 1050 Brussels, Belgium \\ ${ }^{4}$ Laboratory for Cellular and Molecular Immunology, Free University of Brussels (VUB), Pleinlaan 2, 1050 Brussels, Belgium
}

\begin{abstract}
African trypanosomiasis is the collective name for a wide variety of trypanosome infections that affect humans and livestock. In recent years, experimental mice infection models have provided new insights into both human and animal trypanosomiasis. Mouse models seem to be a valuable and versatile tool in trypanosomiasis-associated pathology and immunology research and highlight the variety shown by African trypanosomiases. Indeed, inbred mouse strains have enabled the study of genetic determinants of susceptibility and of the roles of anti-parasite antibodies, inflammatory mediators and anti-inflammatory mediators for each trypanosome species. Remarkable advances relating to the encephalitic stage of sleeping sickness have also been achieved thanks to murine models. The different contributions of murine models to the African trypanosomiases knowledge are presented here. Future search directions are finally proposed, with respect to mouse model opportunities and limitations.
\end{abstract}

\begin{abstract}
'African trypanosomiasis': not a single disease
'African trypanosomiasis' is a parasitic condition that affects both humans and livestock in Sub-Saharan Africa and represents a vital and economic threat. The term 'African trypanosomiasis' actually encompasses different types of diseases and includes infections by a broad range of trypanosome species. Indeed, parasites belonging to the Trypanozoon subgenus - including Trypanosoma brucei rhodesiense and Trypanosoma brucei gambiense (the causative agents of human African trypanosomiasis, or HAT), Trypanosoma brucei brucei, Trypanosoma equiperdum and Trypanosoma evansi (Box 1) - are characterized by their ability to invade the extravascular compartment and to cause the development of neurological disease. In particular, HAT is characterized by two different disease stages. The early stage is characterized by the confined presence of parasites in the blood and lymphoid fluids, whereas the second stage is determined by the entrance of the parasite into the central nervous system (CNS), by breaking through the blood-brain barrier (BBB). By contrast, Trypanosoma congolense (Nanomonas) infections are characterized by a strictly intravascular distribution of
\end{abstract}

Corresponding author: Antoine-Moussiaux, N. (nantoine@ulg.ac.be) the parasite. Trypanosoma vivax (Dutonella) infections constitute a third type of host-pathogen interaction that is poorly characterized.

All African trypanosomes use antigenic variation of their surface antigens as the main defence system against the immune system of their mammalian host; this enables a continuous escape from the host anti-parasite antibody response [1]. The presence of the trypanosome variant surface glycoprotein (VSG) coat, moreover, hampers immunization against the trypanosomes and leaves chemotherapy as the only disease-control method for humans. With respect to animal trypanosomiasis, rearing trypanotolerant cattle might be considered as a sustainable alternative for animal production in glossina-infected areas, where the continuous long-term treatment of animals causes a risk of the induction of drug resistance.

Despite their biological differences, all African trypanosomes cause common pathology signs such as immunosuppression, cachexia and anaemia, for which the underlying mechanisms can vary. Ethical and practical constraints in investigating trypanosomiasis-associated pathology under natural conditions have led, in recent years, to the development of several mouse models. Being highly versatile, the inbred mouse model is a valuable tool in pathology research because it enables genetic studies of particular phenotypic differences between mouse strains and pathogenesis studies by testing the impact on disease outcome of particular treatments on uniform genetic backgrounds. In addition, genotypic alterations (gene knockout or transgenic strains) are now widely used and give this approach a new dimension.

Inbred mice: a tool to dissect natural variations in trypanosomiasis susceptibility

The most frequently used mouse strains for comparison of susceptibility to trypanosomiasis are $\mathrm{C} 57 \mathrm{Bl} / 6, \mathrm{BALB} / \mathrm{c}$ and $\mathrm{C} 3 \mathrm{H} / \mathrm{He}$. Interestingly, despite all possible pitfalls, the susceptibility ranking of these strains is stable among different trypanosome species; $\mathrm{C} 57 \mathrm{Bl} / 6$ mice generally show longer survival and better parasite control than $\mathrm{BALB} / \mathrm{c}$ and $\mathrm{C} 3 \mathrm{H} / \mathrm{He}$ mice [2-8]. However, remarkable differences appear and deserve some attention (Figures $1,2)$. So far, not a single inbred mouse strain is available that can be classified as resistant to trypanosomes (Box 2). 


\section{Box 1. 'African' trypanosomiasis across the world}

Trypanosoma brucei rhodesiense and Trypanosoma brucei gambiense are the causal agents of sleeping sickness and are the only trypanosomes known so far to be directly pathogenic for healthy humans on the African continent. By contrast, whereas Trypanosoma congolense and Trypanosoma vivax are the major agents of Sub-Saharan animal trypanosomiasis, Trypanosoma brucei brucei is also a causal agent. All $T$. brucei species and $T$. congolense parasites are haemoflagellates that are transmitted by Glossina spp. and are, thus, restricted to the distribution area of their vector. However, three other trypanosome species, which are present on the African continent and beyond, are glossina-independent because they are adapted to different transmission pathways. Hence, they can be found outside the tsetse belt. $T$. vivax can be mechanically transmitted and is also present in South America. Similarly, Trypanosoma equiperdum, the agent of dourine in horses, is sexually transmitted and is present in Africa, Europe and Asia. T. evansi is mechanically transmitted by haematophageous flies, such as tabanids, and is widespread across the Sahel and North Africa, Asia and South America, affecting economically important species such as camels, horses and buffaloes. It is interesting to note that in recent years, several cases of human Trypanosoma evansi infections have been reported in India, which were attributed to a lack of apolipoprotein L-1 $[64,65]$.

In T. congolense infection, BALB/c and CBA mice show the most susceptible phenotypes and succumb to infections less than ten days after infection [2]. By contrast, C57Bl/6 mice $T$. congolense infections have been reported to last for over two or four months [2,3]. Survival times below or around 25 days are reported for infections in C3H, CFLP and $\mathrm{A} / \mathrm{J}$ mice $[2,3]$.

In T.b. rhodesiense infections, $\mathrm{C} 3 \mathrm{H} / \mathrm{He}, \mathrm{DBA} / 1, \mathrm{DBA} / 2$ and $\mathrm{BALB} / \mathrm{c}$ mice are described as the most susceptible strains, surviving less than or around 20 days, whereas an intermediate phenotype with survival times between 20 and 30 days is presented by the A/J mouse strain [4-6]. $\mathrm{C} 57 \mathrm{Bl} / 6$ mice also exhibit prolonged survival [4-6]. Similar rankings for $\mathrm{C} 3 \mathrm{H} / \mathrm{He}$ and $\mathrm{C} 57 \mathrm{Bl} / 6$ are obtained in related T.b. brucei infections [2,7]. The CFLP strain has the longest survival for T.b. brucei infections, which greatly differs from $T$. congolense rankings [2]. Interestingly, although the CBA mouse strain was reported to have an intermediate survival phenotype in experimental T.b. rhodesiense infections [4-6], it exhibits prolonged survival in case of $T$. brucei infections, similar to [2] or even longer than C57Bl [7]. These results indicate that besides the genetic background of the host, the precise genetic parasite background and the interaction between the two will also be crucial in determining the lifespan of the infected host. In the same context, it is important to mention that $\mathrm{BALB} / \mathrm{c}$ mice exhibit prolonged survival in T.b. gambiense infection, in contrast to T.b. rhodesiense and T.b. brucei infections, which possibly indicates differences in pathogenesis between the human-infecting subspecies [9].

Susceptibility ranking in $T$. evansi infections has been hampered by the worldwide distribution of this parasite (Box 1) and, thereby, the wide range of parasites used by laboratories. In one study, different rankings resulted from infections with different South American parasite strains [8]. For the six trypanosome strains tested, four presented the classical ranking with $\mathrm{C} 3 \mathrm{H} / \mathrm{He}$ and $\mathrm{BALB} / \mathrm{c}$ showing the shortest survival and $\mathrm{C} 57 \mathrm{Bl} / 6$ the longest. One strain showed inverse phenotypic ranking with $\mathrm{C} 57 \mathrm{Bl} / 6$ being more susceptible than $\mathrm{C} 3 \mathrm{H} / \mathrm{He}$ and $\mathrm{BALB} / \mathrm{c}$ (Figure 2).

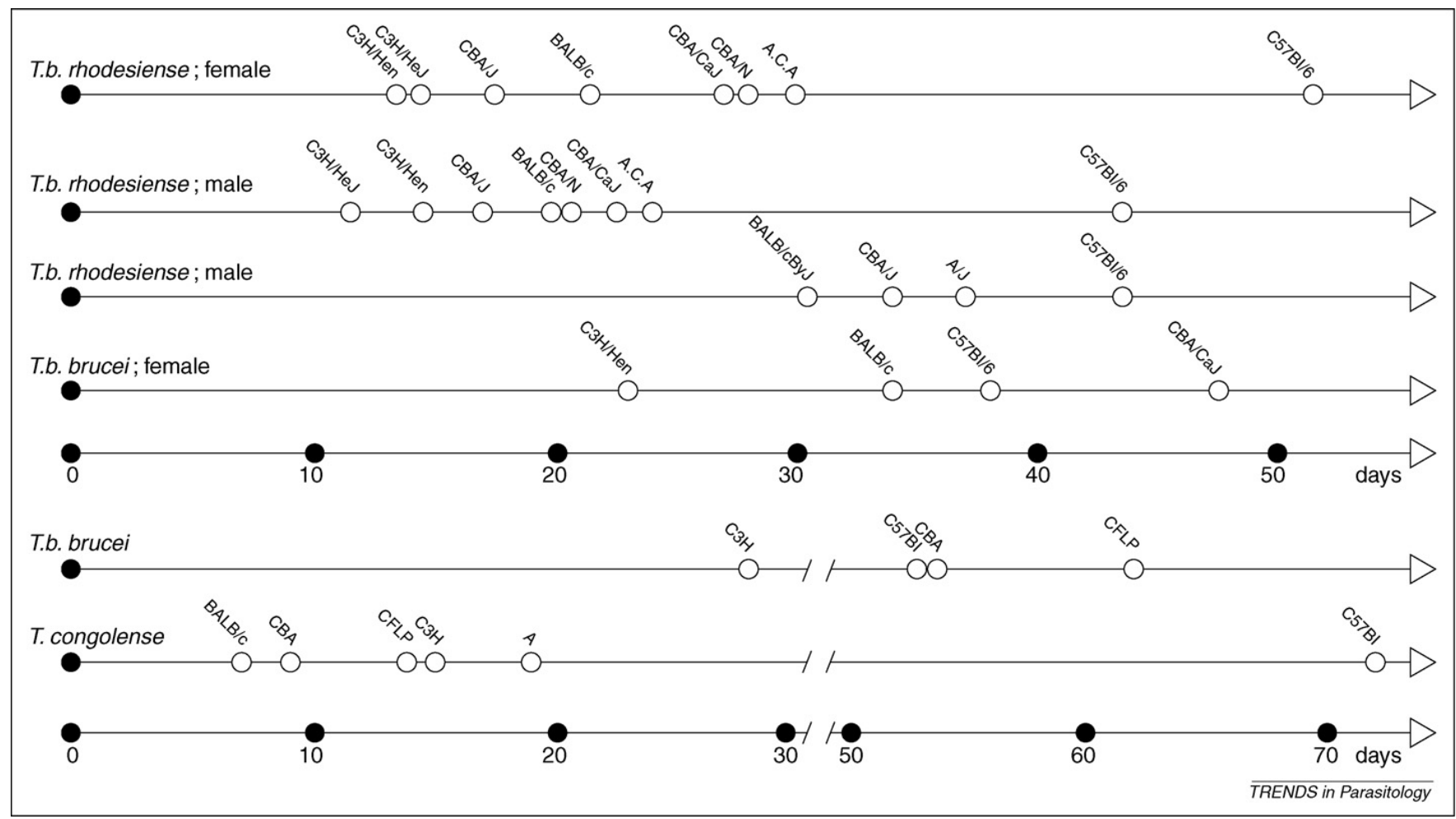

Figure 1. Survival rankings for 'African trypanosomiasis' among different mice strains. This figure illustrates the divergences among mice susceptibility rankings according to the parasite species or strain used. The sex of tested animals is indicated next to the parasite species, when the information was available. Differences in ranking are particularly striking when comparing CBA/CaJ and C57BI/6 between T.b. rhodesiense and T. brucei or C57BI, CBA and CFLP between T. brucei and T. congolense. Data presented in this figure are from Refs $[2,5-7]$. 


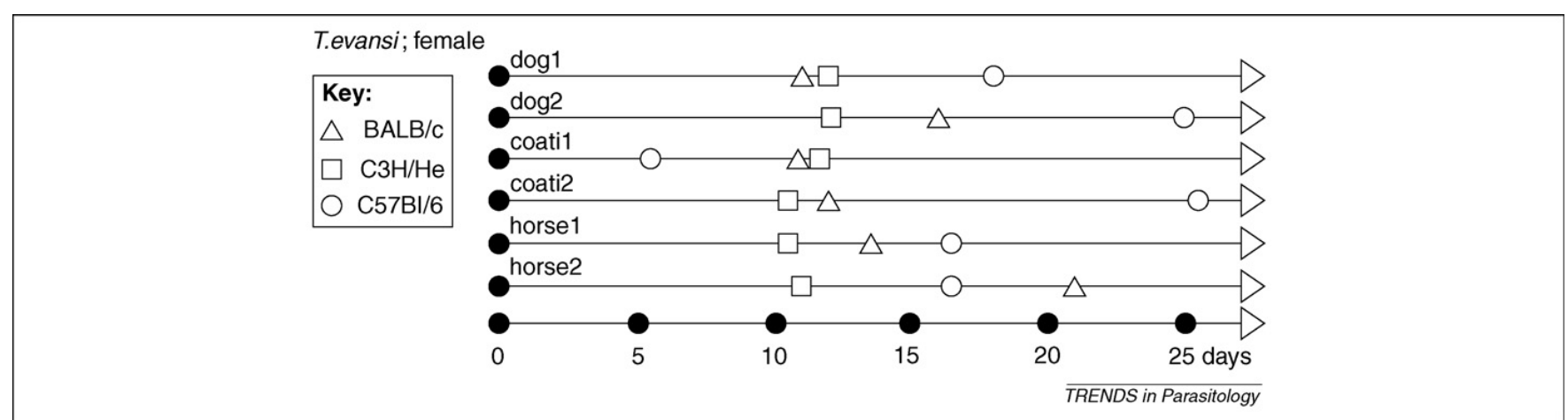

Figure 2. Mice-survival rankings for different $T$. evansi strains. The survival of three classically used mice strains (BALB/c, C3H/He and C57B1/6) after infection with different T. evansi isolates is shown. T. evansi strains are named after the animal species from which they were isolated. Particularly striking are the unusual relative susceptibility of $\mathrm{C} 57 \mathrm{~B} / \mathrm{6}$ to infection by strain 'coati1' and the remarkable relative resistance of BALB/c to infection by strain 'horse2'. Data presented in this figure are from Ref. [8].

\section{Genomics of trypanosomiasis susceptibility: can genetic crossbreeding provide new answers?}

Susceptibility patterns were first used to approach the genetic determinants of murine trypanosomiasis control through breeding schemes. Such breeding schemes involve two mice strains presenting opposite phenotypes and consists of successive crosses. In a first step, they allow the description of the mode of inheritance of the studied phenotype (dominant, recessive, sex-linked or autosomal). Then, through numerous recombinations produced inside the genomes of each obtained individual, a phenotyping procedure coupled to a genomic screening (microsatellites or single nucleotide polymorphism) will enable the study of correlations between phenotype and genotype, further resulting in the detection of quantitative trait loci (QTL) and their mapping.

In $T$. congolense infections, studies of [C57Bl/6xBALB/ c]-crosses indicated that the difference in survival time observed between parental strains was accounted for by three loci [Trypanosoma infection response-1 (Tir1), Tir2 and Tir3, mapped to chromosomes 17, 5 and 1, respectively] [10,11]. Further analysis, using advanced intercross lines (AIL) from $[\mathrm{C} 57 \mathrm{Bl} / 6 \mathrm{xA} / \mathrm{J}]$ and $[\mathrm{C} 57 \mathrm{Bl} / 6 \mathrm{xBALB} / \mathrm{c}]-$ crosses confirmed these three loci and improved resolution

\section{Box 2. Trypanotolerance in mice}

In the past, several authors have used the term 'resistance' to indicate mouse strains that develop slowly progressing infections. However, attention should be paid to the use of words such as 'resistance' versus 'tolerance'. Here, 'tolerance' is considered to be the ability to limit the pathological consequences of infection, and 'resistance' is considered to be the ability to impede the entry or multiplication of an infectious agent, or the complete elimination of an infectious agent from the host. Hence, the term 'resistance' does not apply to murine models for T. brucei, T. congolense, T. evansi or T. vivax because, to date, no mouse strain has been identified that is not killed by any of these parasites. To date, the ranking of mouse strains for susceptibility to trypanosomiasis is made through the follow-up of parasitaemia and survival. However, it should be kept in mind that survival is an imprecise parameter because the cause of death can vary among mouse strains, trypanosome species and disease stages. Indeed, trypanosomiasis-associated death in experimental murine models can result from systemic inflammatory response syndrome (SIRS) $[7,23,26]$, renal failure (possibly linked to immune-complex formation) [8], anaemia or CNS invasion. In addition, immunodepression could make a trypanosome-infected host susceptible to opportunistic infections in situations where mice are exposed to such agents. to a confidence interval of fewer than $20 \mathrm{cM}$ [12]. The role of two Tir1 candidate genes, tumor necrosis factor (TNF) and heat shock protein 70.1 (HSP70.1), has been studied in $T$. congolense-infected $\mathrm{C} 57 \mathrm{Bl} / 6$ mice with deletions in the relevant genes. Although no involvement of HSP70.1 in survival was indicated [13], TNF-disrupted C57Bl/6 mice showed a significantly shortened survival [14,15]. AILanalyses revealed a high degree of complexity of Tir3, which is composed of three loci called 'Tir3a', 'Tir3b' and 'Tir3c'. Marker-assisted introgression of the three loci from a long-surviving donor strain $(\mathrm{C} 57 \mathrm{Bl} / 6)$ to a susceptible recipient strain $(\mathrm{A} / \mathrm{J})$ successfully resulted in a survivaleffect transfer, close to the original mapping population [16]. The cytokine interleukin-10 (IL-10) gene and the Cypr2 locus, which is involved in the regulation of IL-10 synthesis, are candidates for the Tir3b locus [17].

However, early $T$. congolense studies described distinct inheritance modes for survival and parasitaemia control (autosomal dominant and recessive, respectively), thereby indicating that they are independent, as also shown below for T.b. rhodesiense [18,19]. Nevertheless, because these studies do not use the same cross, no conclusion can be drawn because this choice could greatly influence the output [3]. Interestingly, sex-associated factors seemed to confer an advantage in $T$. congolense control to females, as confirmed by more recent studies $[14,19]$.

In contrast to the $T$. congolense-obtained results described above, the results provided by $T$. brucei models are less conclusive. Infection with T.b. rhodesiense LouTat1 in F1 mice (a cross between B10.BR/SgSnJ and $\mathrm{C} 3 \mathrm{HeB} / \mathrm{FeJ}$ ) indicated that survival and parasitaemia control segregate as independent traits, with survival being recessive and parasitaemia control being dominant [20]. These results confirmed an earlier radiation chimaera study that showed independency between both traits [21]. However, a third model, using [C57Bl/6xBALB/c]crosses infected by T.b. rhodesiense EATRO1886, showed survival inherited as a dominant trait [4]. Similar to $T$. congolense infections, females exhibited prolonged survival to T.b. rhodesiense EATRO1886 infection [5]. X-linked genes, however, did not account for this difference.

One consensus that comes out of using different mouse models is the lack of effect of the major histocompatibility complex (MHC), MHC-linked genes or H-2 locus in influencing susceptibility to African trypanosomiasis $[3,6,22]$. 
In conclusion, breeding schemes were successful at providing important clues. As the most conclusive studies studying survival have been done in $T$. congolense, new responses can be expected from the use of different trypanosomes, phenotypes and parental strains. A renewed interest in the long quest to QTL mapping is, thus, needed because this approach has the great advantage of enabling the discovery of unsuspected genes because it is purely phenotype-driven.

\section{Immune responses and trypanosomiasis susceptibility: new answers from the use of knockout mice}

African trypanosomes evade the host immune response through mechanisms that include VSG variation and host immune modulation. The modifications of cytokines, lymphokines and other immune effectors during the course of infection, and their relative role in susceptibility to trypanosomiasis, have been studied in several mouse models. So far, mainly anti-cytokine antibody treatment and knockout mouse models have been used [7,13-15,23-26]. In particular, the use of knockout mice for in vivo studies has recently given trypanosome research a new dimension because antibody-mediated neutralization of immune factors has not always been completely efficient.

\section{Parasite control through anti-VSG antibodies: an operating system that remains to be functionally elucidated}

The main mechanism that is believed to mediate African trypanosomiasis control in a mammalian host is the continuous interplay between the parasite's VSG and host antibodies. Although this interaction is presently considered to be a textbook example, the functional role of the antibodies remains a point of debate in mouse and bovine models [27].

Recently, the role of antibodies has been addressed in experimental models using both B-cell-deficient and immunoglobulin M (IgM)-deficient mice infected with $T$. congolense [28] and T. evansi parasites [29]. In T. congolense, only IgGs were crucial for parasitaemia control [28]. By contrast, in $T$. evansi infections, the main anti-VSG antibody role was executed by the infection-induced IgM antibodies. Interestingly, although the trypanosome-clearing activity of antibodies is most likely due to antibodymediated phagocytosis of opsonized parasites by macrophages, including Kupffer cells [30], there is no experimental data pinpointing the macrophage receptor involved in this process.

A role for complement-mediated lysis in parasite clearance has been proposed but could not be confirmed because T. evansi-infected complement (C5)-deficient AKR mice control successive parasitaemia waves as efficiently as complement-competent strains [29]. In T. brucei infection, although C3 complement factor could aid in hepatic parasite phagocytosis during vaccine-induced parasite clearance, no in vivo contribution of complement-mediated lysis to parasite control could be ascertained [31].

\section{TNF and nitric oxide: the double agents}

Besides antibodies and non-immune effector mechanisms, various immunoregulatory and effector molecules could be involved in trypanosome growth control. In trypanosomiasis, the first cytokine that was described was TNF $[32,33]$. Analysing the possible role of TNF in trypanosomiasis control has confirmed the notion described above that 'African trypanosomiasis' as such is virtually impossible to describe as a single disease. Hence, the review of the data available to date on the role of TNF in trypanosomiasis is presented here in a species-specific manner. The same approach is taken for nitric oxide (NO), an inflammatory mediator that is closely linked to TNF from the point of parasitemia control.

In $T$. congolense infections, TNF-deficient mice present severely shortened survival times and fail to control the first parasitaemia peak $[14,15,34]$. A recent study involving $\mathrm{iNOS}^{-/-}$(inducible nitric oxide synthase-deficient), $\mathrm{TNF}^{-1-}$, TNF-R(receptor) $1^{-/-}$and $\mathrm{TNF}-\mathrm{R} 2^{-/-}$mice confirmed that TNF and NO cooperate in controlling T. congolense [15]. Because T. congolense elimination has been proposed to occur mainly in the liver [30], this study focused on TNF-R1 (CD120a/p55) signal transduction and its role in liver iNOS activation. TNF-R1 signalling was necessary for optimal NO production, NO aided in parasite killing, and the correct anti-VSG antibody induction pathway was activated in the host. Interestingly, NO secretion depended on the Ig isotypes produced; IgG2a induced NO levels that were two- to ninefold higher than those induced by IgM [35].

In T. brucei infections, the role of TNF seems much more complicated because of its possible direct effect on parasite proliferation and its involvement in $T$. brucei-associated anaemia.

TNF induction and the responsible parasite factor (VSG-glycosylphosphatidylinositol anchor) are fully characterized and MyD88 (myeloid differentiation primary response gene 88) dependent [36,37]. During infection, TNF has been reported to be protective in cases in which it exhibits static and lytic effects on the T. brucei parasites [38-40]. In accordance, TNF-deficient mice showed increased parasitaemia in both T.b. brucei and T.b. rhodesiense infection [7,34,41,42]. However, the direct effector function of TNF on trypanosomes is only confirmed in a limited number of $T$. brucei strains and is not a general operating mechanism [43].

Part of $T$. brucei-associated lesions were shown to be TNF-induced through TNF-R2 activation (CD120b/p75) [7]. Indeed, in contrast to mouse strains such as $\mathrm{C} 57 \mathrm{Bl} / 6$ and $\mathrm{C} 3 \mathrm{H} / \mathrm{He}$ that show severe inflammatory-associated pathology during infection, $\mathrm{BALB} / \mathrm{c}$ and $\mathrm{CBA} / \mathrm{Ca}$ mice control inflammation through the shedding of soluble TNF-R2, which could either interrupt receptor signalling or block biological activity through the formation of receptor-ligand complexes [7]. An interesting hypothesis is that TNF-receptor shedding is controlled by IL-10. As is shown below, IL-10 has a crucial role in the establishment of prolonged trypanosome infections.

Finally, in T. evansi models, the role of TNF in parasitaemia control and pathology development seems similar to its role in $T$. brucei models. Indeed, $\mathrm{TNF}^{-/-}$mice show increased parasitaemia, whereas anaemia is better controlled [29]. Again, a crucial role of TNF-R2 has been reported here. 
No role for NO in T. brucei control could be established through knockout mice studies; $\mathrm{iNOS}^{-/-}$mice behaved similarly to fully immune-competent control mice [4446]. However, treatment studies using a specific NOS inhibitor (L-NAME), in addition to one knockout-mice study, highlighted the role of NO in T. brucei-induced immunosuppression and anaemia [46-48]. This illustrates the complementarities of knockout mice and treatment studies: both differ in the specificity of inhibition and, thus, in the importance of the redundancy phenomenon, which refers to the take-over of the removed cytokine or protein function by present cytokines or proteins.

These data once again stress the difference between the immune control of the intravascular $T$. congolense parasites and the more systemic control needed in case of $T$. brucei or T. evansi infection (Figure 3).

\section{Interferon- $\gamma$ and IL-10: the vital balance}

Besides TNF and NO, several additional host immune factors determine the development of parasitaemia and infection-associated pathology.

In $T$. congolense infections, the need for an early inflammatory response in efficient parasite control explains the observed lack of proper parasitemia control and early death of infected $\mathrm{C} 57 \mathrm{Bl} / 6$ interferon (IFN)- $\gamma^{-/-}$mice [28]. However, results obtained with IL- $10^{-1-}$ mice show that once an effective inflammation has been mounted, an IL-10-driven response is needed to limit inflammationinduced lesions [49]. In this regard, IFN- $\gamma$ has been described as a susceptibility factor in a BALB/c T. congolense model characterized by early host death, and a link has been established between early death and unrestrained IFN- $\gamma$ production that results in an unbalanced IFN- $\gamma$-IL-10 response [26]. T. congolense infections in IFN- $\gamma^{-/-} \mathrm{C} 57 \mathrm{Bl} / 6$ and BALB/c mice that were treated with anti-IFN- $\gamma$ antibodies highlighted these different aspects of IFN- $\gamma$ involvement as a result of different genetic backgrounds [25,28,45].

In T. brucei infections, similar IFN- $\gamma$ and IL-10 roles are described. Indeed, whereas IFN $-\gamma^{-1-}$ mice fail to clear parasitaemia and succumb to uncontrolled parasite growth, IL- $10^{-/-}$mice control parasitaemia as efficiently as wild-type mice but succumb because of hyperacute inflammation after the first parasitemia peak [50]. Hence, a chronic $T$. brucei model (phospholipase-C-deleted parasites, or $\mathrm{PLC}^{-/-}$) showed the conversion of an early-stage IFN- $\gamma$ inflammatory response to a late-stage IL-10-dominated response to coincide with prolonged survival [51].

Combined, these studies point out the central importance of the IFN- $\gamma$-to-IL-10 ratio for controlling parasitaemia with minimal host damage in both $T$. congolense and $T$. brucei infections. Although scarce, data about the cellular source of IFN- $\gamma$ and IL-10 seem to be model-dependent. Whereas Shi et al. [26] showed a role for $\mathrm{CD} 4^{+} \mathrm{T}$ cells in the secretions of both cytokines, Namangala et al. [50] showed IFN- $\gamma$ to be produced mainly by CD8 ${ }^{+} \mathrm{T}$ cells and IL-10 to be partially secreted by $\mathrm{CD}^{+} \mathrm{T}$ cells. These divergences possibly relate to differences of the parasite or the mouse strain studied. Recently, the induction of IL-10-producing regulatory $\mathrm{T}$ cells in $T$. congolense infection was also reported to have a major role in the inhibition of excessive infection-associated inflammation [52]. However, no regulatory T-cell induction has been reported in T. brucei infections, despite the crucial role that IL-10 also has there.

\section{The encephalitic stage of trypanosomiasis: the problem that kills}

As mentioned earlier, trypanosome species such as $T$. brucei, T.b. rhodesiense and T.b. gambiense are able to cross the BBB, causing severe neurological disorders and risking post-treatment reactive encephalopathy (PTRE); this has been reviewed recently by others [53-55].

PTRE relates to an exacerbation of the clinical and pathological features of CNS involvement that is observed after treatment with an arsenical compound, such as melarsopol, in $\sim 10 \%$ of patients [53]. In mice, the 'PTRE model' is an infection-treatment model that dynamically reveals different grades of pathogeny: mild pathogenic CNS invasion, medium-to-severe pathogenic CNS invasion (after one trypanolytic treatment) and severe PTRE (after a second treatment) [56]. Analysis of this model has shown a pivotal role for astrocyte activation, as well as the importance of endogenous anti-inflammatory cytokines (IL-10 and IL-6), in the alleviation of neuropathogenesis during early CNS invasion, and the later involvement of endogenous TNF in severe cases [56,57]. Other important factors in this neuropathogenic process were autoantibodies directed against myelin basic protein, myelinspecific galactocerebrosides and gangliosides, in addition to substance P (SP), the role of which was highlighted through in vivo neutralization assays and knockout-mice studies [58-61]. Again, differences between results obtained in both types of studies illustrate the difficulties in addressing the redundancy problem.

Mouse BBB-crossing models have been crucial in demonstrating the central role of laminin composition of the basement membrane of the microvascular endothelial cells, as well as the role of IFN- $\gamma$ in the migration process of trypanosomes through the BBB [62]. This recent research involved several knockout mice strains, namely IFN- $\gamma^{-1-}$, IFN- $\gamma$ receptor $^{-/-}$, p40 subunit of IL-12 $2^{-/-}$(IL-12p40 ${ }^{-/-}$) and recombinant activating gene- $1^{-/-}\left(\mathrm{RAG}^{-1^{--}}\right)$. In addition, further treatment studies showed trypanosome migration to be impeded by Minocyclin, a tetracycline antibiotic that impedes leucocyte migration in the CNS in experimental allergic encephalitis [63].

\section{Concluding remarks: where to go from here?}

Mouse models have proven to be a valuable tool in understanding trypanosomiasis susceptibility and pathogenesis. This is due to the high variability of phenotypes that are presented by inbred strains and the powerful investigation tools readily available for murine studies. In the future, full advantage should be taken of the different genetic backgrounds in studies using gene deletion, monoclonal antibody or cytokine treatment to solve the many remaining questions that surround trypanosomiasis pathogenesis and the development of new treatment modalities. Although significant progress has been made in trypanosome immunopathology research in recent years, other topics remain poorly understood. Indeed, it remains to be fully discovered (i) how parasitemia growth control is 


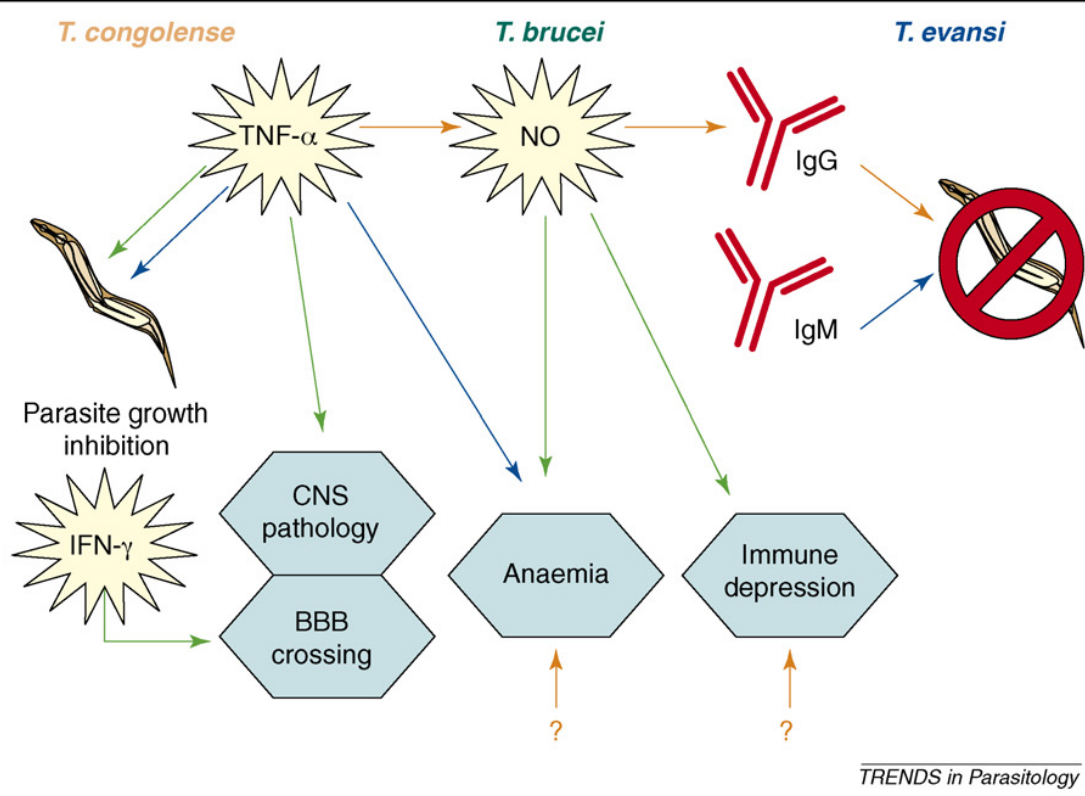

Figure 3. Diagram of major differences among known pathogenesis and defence mechanisms for different 'African trypanosomiasis' agents. The most remarkable differences in trypanosome pathogenesis and host defence mechanisms are schematically represented for $T$. congolense (orange arrows), T. brucei (green arrows) and $T$. evansi (blue arrows). These include: (i) the absence of involvement of TNF or NO in anaemia and immune-depression development, and the absence of BBB crossing and CNS pathology for the strictly intravascular T. congolense compared with the major pathological role of TNF, NO and interferon- $\gamma$ (IFN- $\gamma$ ) in $T$. brucei infection; (ii) the direct effect of TNF on $T$. brucei growth but lack of major involvement of TNF in global defence against $T$. brucei and $T$. evansi in contrast to $T$. congolense; (iii) and the preponderance of $\lg \mathrm{M}$ in $T$. evansi clearance compared to the pivotal role of IgG in $T$. congolense infection (see text for detailed mechanisms).

regulated in vivo, (ii) how trypanosomiasis-associated anaemia is induced, (iii) how the regulation of cytokine balance occurs throughout infection and (iv) what the molecular and cellular mechanisms are that underlie the BBB passage of parasites. These are only a few funda-

\section{Box 3. From mouse to cattle and man?}

Undoubtedly, differences occur between long-term murine survival models and bovine trypanotolerance. Indeed, bovine trypanotolerance (as in the N'Dama breed) consists of efficient parasite control and reduced lesions, whereas in murine survival models (which are classically represented by C57BI/6 mice), parasite clearance is accompanied by enhanced lesions. However, this must not be considered as an obstacle to studying trypanotolerance through mouse models because their advantage interest lies in the high variability of phenotypes presented by inbred strains. Examples of successful parallels between murine model breakthroughs and bovine pathology have been reported in the context of the role of TNF and for the role of anti-VSG Ig in parasite control. Studies conducted in knockout mice (see the main text) relate to the finding in a chimaera bovine-infection model that $T$. congolense susceptibility and parasitaemia control are regulated independently from the haematopoietic system [27] and to the reported correlation between trypanotolerance in livestock and increased specific $\lg G$ production $[66,67]$.

Host genotype influence in HAT is suspected from the wide variations in disease severity, as recently shown in populations from Uganda, Zimbabwe and Malawi, reminiscent of earlier propositions for innate resistance in Bantu populations [68]. Interestingly, transforming growth factor- $\beta$ involvement in Malawian subtolerance cases has been suspected, possibly through the limitation of TNF secretion and, thus, TNF-mediated pathogenesis. A recent study using single nucleotide polymorphisms in TNF and IL-10 genes and promoter loci indicates a protective role for a particular IL-10 mutation (IL-10-592a) in resistance to HAT, whereas a TNF- $\alpha-308$ promoter mutation correlated with a higher risk of developing disease early after exposure [69]. Hence, as in mice, it seems that HAT pathogenesis is crucially influenced by the balance between TNF and IL-10. mental topics that can be addressed using comparative studies in different mouse models, supplemented with studies in gene-deficient mice. These studies combined should then provide new methods to combat both HAT and livestock trypanosomiasis (Box 3).

\section{Acknowledgements}

Many thanks are due to $\mathrm{P}$. Büscher for his valuable advice during the early development of the present article.

\section{References}

1 McCulloch, R. (2004) Antigenic variation in African trypanosomes: monitoring progress. Trends Parasitol. 20, 117-121

2 Jennings, F.W. et al. (1978) The susceptibility of strains of mice to infection with Trypanosoma congolense. Res. Vet. Sci. 25, 399-400

3 Morrison, W.I. and Murray, M. (1979) Trypanosoma congolense: inheritance of susceptibility to infection in inbred strains of mice. Exp. Parasitol. 48, 364-374

4 Greenblatt, H.C. et al. (1984) Trypanosoma rhodesiense: analysis of the genetic control of resistance among mice. Infect. Immun. 44, 107-111

5 Greenblatt, H.C. and Rosenstreich, D. (1984) Trypanosoma rhodesiense infection in mice: sex dependence of resistance. Infect. Immun. 43, 337340

6 Levine, R.F. and Mansfield, J.M. (1981) Genetics of resistance to African trypanosomes: role of the H-2 locus in determining resistance to infection with Trypanosoma rhodesiense. Infect. Immun. 34, 513-518

7 Magez, S. et al. (2004) P75 tumor necrosis factor-receptor shedding occurs as a protective host response during African trypanosomiasis. J. Infect. Dis. 189, 527-539

8 De Menezes, V.T. et al. (2004) Trypanosoma evansi in inbred and SwissWebster mice: distinct aspects of pathogenesis. Parasitol. Res. 94 193-200

9 Inoue, N. et al. (1999) Interleukin 4 is a crucial cytokine in controlling Trypanosoma brucei gambiense infection in mice. Vet. Parasitol. 86, 173-184

$10 \mathrm{Kemp}$, S.J. et al. (1996) Genetic control of resistance to trypanosomiasis. Vet. Immunol. Immunopathol. 54, 239-243 
11 Kemp, S.J. et al. (1997) Localization of genes controlling resistance to trypanosomiasis in mice. Nat. Genet. 16, 194-196

12 Iraqi, F. et al. (2000) Fine mapping of trypanosomiasis resistance loci in murine advanced intercross lines. Mamm. Genome 11, 645-648

13 Nakamura, Y. et al. (2003) Susceptibility of heat shock protein 70.1deficient $\mathrm{C} 57 \mathrm{BL} / 6 \mathrm{~J}$, wild-type $\mathrm{C} 57 \mathrm{BL} / 6 \mathrm{~J}$ and $\mathrm{A} / \mathrm{J}$ mice to Trypanosoma congolense infection. Parasitol. Res. 90, 171-174

14 Iraqi, F. et al. (2001) Susceptibility of tumour necrosis factor- $\alpha$ genetically deficient mice to Trypanosoma congolense infection. Parasite Immunol. 23, 445-451

15 Magez, S. et al. (2007) Tumor necrosis factor (TNF) receptor-1 (TNFp55) signal transduction and macrophage-derived soluble TNF are crucial for nitric oxide-mediated Trypanosoma congolense parasite killing. J. Infect. Dis. 196, 954-962

16 Koudande, O.D. et al. (2005) Marker-assisted introgression of trypanotolerance QTL in mice. Mamm. Genome 16, 112-119

17 Tabel, H. et al. (2000) Susceptibility and resistance to Trypanosoma congolense infections. Microbes Infect. 2, 1619-1629

18 Morrison, W.I. et al. (1979) Genetic control of susceptibility to T. congolense infection in inbred strains of mice. Adv. Exp. Med. Biol. 114, 709-714

19 Pinder, M. (1984) Trypanosoma congolense: genetic control of resistance to infection in mice. Exp. Parasitol. 57, 185-194

20 De Gee, A.L. et al. (1988) Genetics of resistance to the African trypanosomes. VI. Heredity of resistance and variable surface glycoprotein-specific immune responses. J. Immunol. 140, 283-288

21 De Gee, A.L. and Mansfield, J. (1984) Genetics of resistance to the African trypanosomes. IV. Resistance of radiation chimaeras to Trypanosoma rhodesiense infection. Cell. Immunol. 87, 85-91

22 Levine, R.F. and Mansfield, J. (1984) Genetics of resistance to the African trypanosomes. III. Variant-specific antibody responses of H-2compatible resistant and susceptible mice. J. Immunol. 133, 15641569

23 Shi, M. et al. (2003) Experimental African trypanosomiasis: IFN- $\gamma$ mediates early mortality. Eur. J. Immunol. 33, 108-118

24 Uzonna, J.E. et al. (1998) Experimental murine Trypanosoma congolense infections. I. Immunoregulation in experimental murine Trypanosoma congolense infection: anti-IL-10 antibodies reverse trypanosome-mediated suppression of lymphocyte proliferation in vitro and moderately prolong the lifespan of genetically susceptible BALB/c mice. Parasite Immunol. 20, 293-302

25 Uzonna, J.E. et al. (1998) Experimental murine Trypanosoma congolense infections. I. Administration of anti-IFN-gamma antibodies alters trypanosome-susceptible mice to a resistant-like phenotype. J. Immunol. 161, 5507-5515

26 Shi, M. et al. (2006) Experimental African trypanosomiasis: a subset of pathogenic, IFN- $\gamma$-producing, MHC class II-restricted $\mathrm{CD}^{+}{ }^{+} \mathrm{T}$ cells mediates early mortality in highly susceptible mice. J. Immunol. 176, 1724-1732

27 Naessens, J. et al. (2003) Responses of bovine chimaeras combining trypanosomosis resistant and susceptible genotypes to experimental infection with Trypanosoma congolense. Vet. Parasitol. 111, 125-142

28 Magez, S. et al. (2006) Interferon- $\gamma$ and nitric oxide in combination with antibodies are key protective host immune factors during Trypanosoma congolense Tc13 infections. J. Infect. Dis. 193, 1575-1583

29 Baral, T.N. et al. (2007) Control of Trypanosoma evansi infection is IgM mediated and does not require a type I inflammatory response. J. Infect. Dis. 195, 1513-1520

30 Shi, M. et al. (2004) Trypanosoma congolense infections: antibodymediated phogocytosis by Kupffer cells. J. Leukoc. Biol. 76, 399-405

31 Macaskill, J.A. et al. (1980) Immunological clearance of $75^{\mathrm{Se}}$-labelled Trypanosoma brucei in mice. Immunology 40, 629-635

32 Hotez, P.J. et al. (1984) Lipoprotein lipase suppression in 3T3-L1 cells by a haematoprotozoan-induced mediator from peritoneal exudate cells. Parasite Immunol. 6, 203-209

33 Beutler, B. et al. (1985) Identity of tumour necrosis factor and the macrophage-secreted factor cachectin. Nature 316, 552-554

34 Naessens, J. et al. (2005) TNF- $\alpha$ mediates the development of anaemia in a murine Trypanosoma brucei rhodesiense infection, but not the anaemia associated with a murine Trypanosoma congolense infection. Clin. Exp. Immunol. 139, 405-410

35 Kaushik, R.S. et al. (1999) Innate resistance to Trypanosoma congolense infections: differential production of nitric oxide by macrophages from susceptible $\mathrm{BALB} / \mathrm{c}$ and resistant $\mathrm{C} 57 \mathrm{Bl} / 6$ mice. Exp. Parasitol. 92, 131-143

36 Magez, S. et al. (1998) The glycosyl-inositol-phosphate and dimyristoylglycerol moieties of the glycosylphosphatidylinositol anchor of the trypanosome variant-specific surface glycoprotein are distinct macrophage-activating factors. J. Immunol. 160, 1949-1956

37 Drennan, M.B. et al. (2005) The induction of a type 1 immune response following a Trypanosoma brucei infection is MyD88 dependent. J. Immunol. 175, 2501-2509

38 Lucas, R. et al. (1994) Mapping the lectin-like activity of tumor necrosis factor. Science $263,814-817$

39 Magez, S. et al. (1997) Specific uptake of tumor necrosis factor- $\alpha$ is involved in growth control of Trypanosoma brucei. J. Cell Biol. 137, $715-727$

40 Magez, S. et al. (1993) Murine tumour necrosis factor plays a protective role during the initial phase of the experimental infection with Trypanosoma brucei brucei. Parasite Immunol. 15, 635-641

41 Magez, S. et al. (1999) Tumor necrosis factor $\alpha$ is a key mediator in the regulation of experimental Trypanosoma brucei infections. Infect. Immun. 67, 3128-3132

42 Magez, S. et al. (2002) Control of experimental Trypanosoma brucei infections occurs independently of lymphotoxin- $\alpha$ induction. Infect. Immun. 70, 1342-1351

43 Kitani, H. et al. (2002) Recombinant tumor necrosis $\alpha$ does not inhibit the growth of African trypanosomes in axenic cultures. Infect. Immun. $70,2210-2214$

44 Chiejina, S. et al. (2003) Concurrent infections with Trypanosoma brucei and Nippostrongylus brasiliensis in mice deficient in inducible nitric oxide. Parasitol. Int. 52, 107-115

45 Hertz, C.J. and Mansfield, J. (1999) IFN- $\gamma$-dependent nitric oxide production is not linked to resistance in experimental African trypanosomiasis. Cell. Immunol. 192, 24-32

46 Millar, A.E. et al. (1999) T-cell responses during Trypanosoma brucei infections in mice deficient in inducible nitric oxide synthase. Infect. Immun. 67, 3334-3338

47 Sternberg, J. et al. (1994) Inhibition of nitric oxide synthesis leads to reduced parasitaemia in murine Trypanosoma brucei infection. Infect. Immun. 62, 2135-2137

48 Mabbott, N. and Sternberg, J. (1995) Bone marrow nitric oxide production and development of anaemia in Trypanosoma bruceiinfected mice. Infect. Immun. 63, 1563-1566

49 Noel, W. et al. (2002) Infection stage-dependent modulation of macrophage activation in Trypanosoma congolense-resistant and susceptible mice. Infect. Immun. 70, 6180-6187

50 Namangala, B. et al. (2001) Relative contribution of interferon- $\gamma$ and interleukin-10 to resistance to murine African trypanosomosis. J. Infect. Dis. 183, 1794-1800

51 Namangala, B. et al. (2001) Alternative versus classical macrophage activation during experimental African trypanosomosis. Int. $J$. Parasitol. 31, 575-587

52 Guilliams, M. et al. (2007) African trypanosomiasis: naturally accruing regulatory T-cells favor trypanotolerance by limiting pathology associated with sustained type 1 inflammation. J. Immunol. 179, $2748-2757$

53 Kennedy, P.G. (2006) Diagnostic and neuropathogenesis issues in human African trypanosomiasis. Int. J. Parasitol. 36, 505-512

54 Kennedy, P.G. (2007) Animal models of human African trypanosomiasis: very useful or too far removed? Trans. R. Soc. Trop. Med. Hyg. 101, 1061-1062

55 Masocha, W. et al. (2007) Migration of African trypanosomes across the blood-brain barrier. Physiol. Behav. 92, 110-114

56 Sternberg, J.M. et al. (2005) Meningoencephalitic African trypanosomiasis: brain IL-10 and IL- 6 are associated with protection from neuro-inflammatory pathology. J. Neuroimmunol. 167, 81-89

57 Hunter, C.A. et al. (1992) Astrocyte activation correlates with cytokine production in central nervous system of Trypanosoma brucei bruceiinfected mice. Lab. Invest. 67, 635-642

58 Eckersall, P.D. et al. (2001) Cytokines and the acute phase response in post-treatment reactive encephalopathy of Trypanosoma brucei brucei infected mice. Parasitol. Int. 50, 15-26

59 Hunter, C.A. et al. (1992) Correlation of autoantibody titres with central nervous system pathology in experimental African trypanosomiasis. J. Neuroimmunol. 41, 143-148 
60 Kennedy, P.G. et al. (1997) A substance P antagonist, RP-67,580, ameliorates a mouse meningoencephalitic response to Trypanosoma brucei brucei. Proc. Natl. Acad. Sci. U. S. A. 94, 4167-4170

61 Kennedy, P.G. et al. (2003) Clinical and neuroinflammatory responses to meningoencephalitis in substance $\mathrm{P}$ receptor knockout mice. Brain $126,1683-1690$

62 Masocha, W. et al. (2004) Cerebral vessel laminins and IFN- $\gamma$ define Trypanosoma brucei brucei penetration of the blood-brain barrier. J. Clin. Invest. 114, 689-694

63 Masocha, W. et al. (2006) Minocycline impedes African trypanosome invasion of the brain in a murine model. Antimicrob. Agents Chemother. 50, 1798-1804

64 Joshi, P.P. et al. (2005) Human trypanosomiasis caused by Trypanosoma evansi in India: the first case report. Am. J. Trop. Med. Hyg. 73, 491-495
65 Vanhollebeke, B. et al. (2006) Human Trypanosoma evansi infection linked to a lack of apolipoprotein L-1. N. Engl. J. Med. $355,2752-2756$

66 Taylor, K. et al. (1996) Nitric oxide synthesis is depressed in Bos indicus cattle infected with Trypanosoma congolense and Trypanosoma vivax and does not mediate T-cell suppression. Infect. Immun. 64, 4115-4122

67 Williams, D.J. et al. (1996) The role of anti-variable surface glycoprotein antibody responses in bovine trypanotolerance. Parasite Immunol. 18, 209-218

68 Sternberg, J.M. (2004) Human African trypanosomiasis: clinical presentation and immune response. Parasite Immunol. 26, 469-476

69 Courtin, D. et al. (2006) Interest of tumor necrosis factor- $\alpha-308$ G/A and interleukin-10-2 C/A polymorphisms in human African trypanosomiasis. Infect. Genet. Evol. 6, 123-129 